\title{
Improving inpatient cystic fibrosis pulmonary exacerbation care: two success stories
}

\author{
Nicholas J Antos, ${ }^{1}$ Diana R Quintero, ${ }^{1}$ Christine M Walsh-Kelly, ${ }^{2}$ \\ Julie E Noe, ${ }^{1}$ Michael S Schechter ${ }^{3}$
}

\begin{abstract}
${ }^{1}$ Division of Pulmonary and Sleep Medicine, Department of Pediatrics, Medical College of Wisconsin, Children's Hospital of Wisconsin, Milwaukee, Wisconsin, USA

${ }^{2}$ Department of Pediatrics, University of North Carolina, Chapel Hill, North Carolina, USA ${ }^{3}$ Department of Pediatrics, Virginia Commonwealth University, Richmond, Virginia, USA
\end{abstract}

\section{Correspondence to} Dr Nicholas J Antos, Department of Pediatrics, Division of Pulmonary and Sleep Medicine, Medical College of Wisconsin, Children's Hospital of Wisconsin, 9000 W Wisconsin Avenue, PO Box 1997, Suite B620, Milwaukee, WI 53201, USA; nantos@mcw.edu

Received 26 July 2013 Revised 25 October 2013 Accepted 4 December 2013

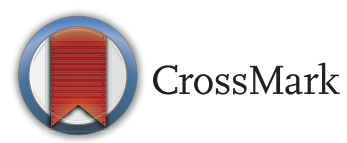

To cite: Antos NJ, Quintero DR, Walsh-Kelly CM, et al. BMJ Qual Saf 2014;23: i33-i41.

\begin{abstract}
Background Pulmonary exacerbations (PEx) in cystic fibrosis (CF) are a frequent cause of hospitalisations and lead to long-term decline in pulmonary function. Successful CF inpatient care requires the coordination of multiple providers and complex therapies. Children's Hospital of Wisconsin (CHW) and Children's Healthcare of Atlanta (CHoA) independently identified PEx inpatient care for focused improvements, with emphasis on improving care coordination and patient outcomes.

Methods Both centres began by forming multidisciplinary workgroups, including patient and family representatives. CHW's specific aim was to eliminate delays in the time to initial intravenous antibiotics. A written handoff tool was developed to allow more efficient ordering. Efforts at CHoA focused on coordination and consistent care delivery. A written schedule and patient incentive programme were devised to ensure proper administration of treatments and promote patient adherence.
\end{abstract}

Results At CHW, interventions decreased the mean antibiotic order time by $59 \%$ with resultant decrease in administration time by $25 \%$. At $\mathrm{CHoA}$, improvements led to a $42 \%$ decrease in the proportion of hospitalisations unsuccessful in returning lung function back to within $90 \%$ of baseline.

Conclusions Inpatient CF PEx care is complex and requires multiple competing activities and treatments. Consistent and timely delivery of these treatments is challenging. Our improvements used the skills and insights of providers and patients to improve, standardise and synchronise care, and to develop tools to coordinate hand offs. With these improvements, applicable to hospital treatment of many other conditions, both centres were successfully able to deliver treatments in a more consistent and timely manner with improved outcomes.

\section{BACKGROUND}

Treatment of pulmonary exacerbations (PEx) represents a significant burden in cystic fibrosis (CF) care. $^{1-4} \mathrm{CF}$ registry data shows that about a third of all patients are treated for at least one PEx each year. ${ }^{1}$ Treatment is often unsuccessful at restoring pulmonary function back to baseline values, and exacerbations are important contributors to the long-term decline in lung function. ${ }^{5} 6$ Most PEx treatment is delivered in the hospital, with an average inpatient stay of 10 days. $^{1}$ These hospitalisations represent a chaotic and stressful time for patients with $\mathrm{CF}$ and their families. ${ }^{2} 7$ Symptoms affect many systems and nutritional concerns often accompany pulmonary symptoms. ${ }^{4}$ Inpatient treatment plans are complex, and include multiple intravenous antibiotics, aggressive airway clearance therapy (ACT) and nutritional rehabilitation regimens. ${ }^{4} 8$ There is no standardised treatment protocol and much variation exists in treatment strategies. ${ }^{8}$ The complexity and variety of these treatments leads to significant delay, missed treatments and difficulties in coordination of care.

Although substantial efforts have been devoted to improving the quality of outpatient CF care, ${ }^{9}$ little has been written on improving inpatient PEx treatment. However, sizeable literature exists in a variety of other contexts describing the positive impact of making systems-based improvements in hospital care. ${ }^{10-12}$ It is reasonable to believe that similar improvements in treatment processes and outcomes can be achieved in the treatment of CF PEx. This paper describes initiatives to improve the quality of $\mathrm{CF}$ pulmonary exacerbation care which were initiated separately at Children's Hospital 
of Wisconsin (CHW) in Milwaukee, Wisconsin and at Children's Healthcare of Atlanta (CHoA) in Atlanta, Georgia with similar strategies for improvement.

\section{CHW INITIATIVE TO IMPROVE THE TIMELINESS AND WORKFLOW OF INITIAL ANTIBIOTIC TREATMENT}

\section{Context}

The paediatric $\mathrm{CF}$ programme at $\mathrm{CHW}$ cares for about 230 patients. Located in Milwaukee, Wisconsin, it is a large academic facility associated with the Medical College of Wisconsin. There are 80-100 CF PEx admissions at this site each year.

Parents at our CF centre voiced concerns regarding the delay in initiating intravenous antibiotics upon admission to the hospital. Most families perceive intravenous antibiotics as the main reason for hospitalisation and this delay added to the concerns and stresses of hospital admission. Improvement efforts were therefore focused on antibiotic timeliness, with a specific aim to decrease the time from arrival on the inpatient unit until the initial antibiotic administration.

\section{Methods and interventions}

Two teams were assembled to oversee quality improvement (QI) efforts: a multidisciplinary steering committee, consisting of inpatient and outpatient staff, and a patient advisory board, including patients and families with admission experience. To assess our baseline state, we mapped our full process from the time a PEx is recognised until the first inpatient antibiotic is administered, as shown in figure 1A. Notably, although the choice of initial antibiotic was made very early in the process, it was not until the last resident house staff step that these orders were actually placed. With multiple associated hand offs, redundant steps and competition for access to the patient there was significant delay before the ordering step. Improving the time to placement of orders in the computerised medical record was therefore chosen for initial improvement focus.

In planning the intervention, our goal was to move the order placement step as early as possible in the house staff workflow without compromising safety or trainee education. As an academic institution, the admission process at $\mathrm{CHW}$ includes an evaluation by multiple trainees and callbacks to discuss plans with supervising physicians. The roadblocks to timely administration were many and related to: (A) handoff schemes, (B) resident workflow and (C) staffing methods. To address these concerns, we created a written handoff tool to aid our verbal sign-out. The tool was revised and edited in multiple versions and incorporated as part of a larger CF Admission Road Map developed at our centre.

The handoff tool contains basic patient information, reasons for admission, and initial antibiotic and ACT plan. It is completed by the outpatient physician when a PEx is recognised in clinic and transferred to the inpatient providers downstream. The use of this written form allows the residents to safely initiate the appropriate antibiotics before general patient discussions and without a redundant check back with supervising physicians. The resultant workflow (figure 1B) allows for immediate order placement upon arrival. A call back is retained as a later step to review all systems, discuss the long-term plan, and educate the team. The tool was initially trialled intradepartmentally for feasibility with success. Before general implementation, multiple training sessions were held for primary CF providers, the entire pulmonary team, and all paediatric house staff, among others. Discipline-specific interventions and inputs are detailed in table 1.

Outcomes of the interventions were studied using a time series design. Improvements were monitored using statistical process control charts, with control limits at three SDs. A shift of eight or more points
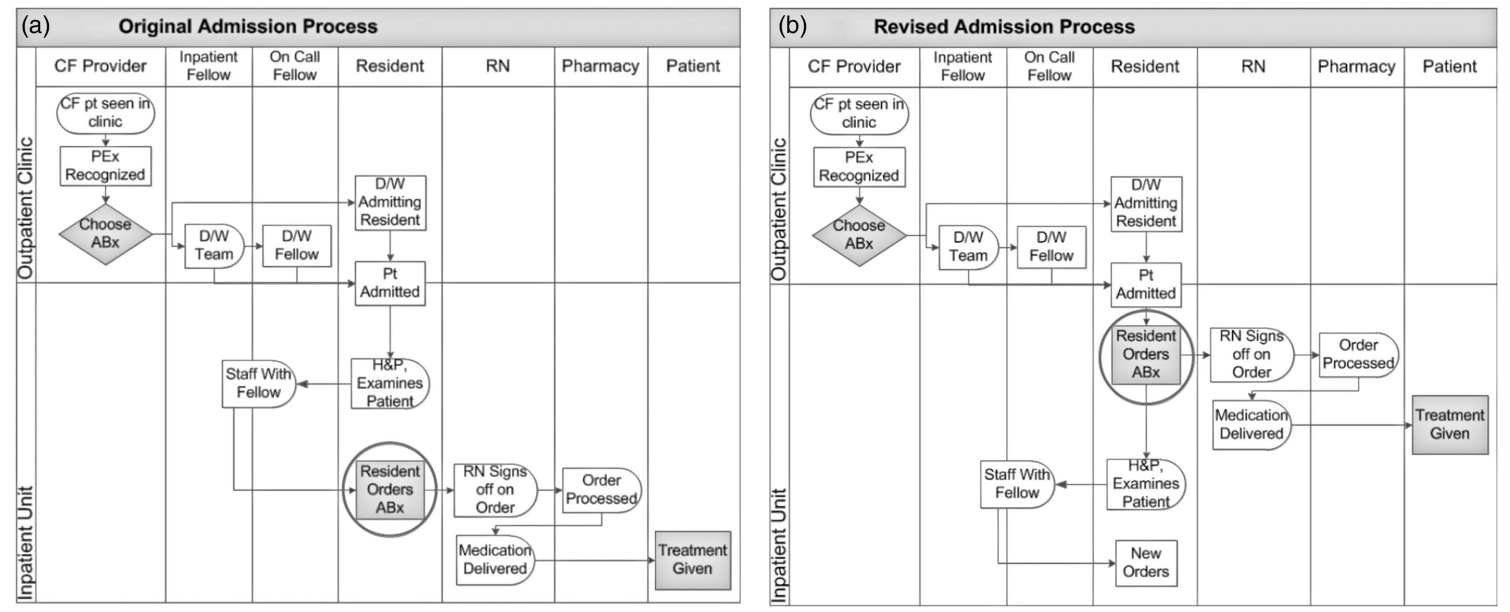

Figure 1 Deployment process maps showing initial process $(A)$ and revised process after intervention (B). Significant antibiotic order and administration steps are shaded, with order placement circled. ABx, antibiotic; CF, cystic fibrosis; DM, discuss with; H\&P, history and physical; PEx, pulmonary exacerbations; RN, nurse. 
Table 1 Improvement steps at Children's Hospital of Wisconsin

\begin{tabular}{|c|c|c|}
\hline & Initial intervention & Subsequent PDSA cycles \\
\hline $\begin{array}{l}\text { Multidisciplinary } \\
\text { team }\end{array}$ & $\begin{array}{l}\text { Examined and mapped workflow } \\
\text { Evaluated baseline timeliness } \\
\text { Created written handoff tool } \\
\text { - Revised tool for format and usefulness }\end{array}$ & $\begin{array}{l}\text { - Monitored measures and adherence } \\
\text { - Revised tool for usability based on feedback } \\
\text { - Created electronic version of tool }\end{array}$ \\
\hline Patients and families & $\begin{array}{l}\text { Voiced initial concerns } \\
\text { Participated in focus groups on patient experience, concerns } \\
\text { and barriers }\end{array}$ & $\begin{array}{l}\text { Provided general feedback } \\
\text { Participated in focus groups on new process }\end{array}$ \\
\hline CF centre staff & $\begin{array}{l}\text { Trialled tool } \\
\text { Identified barriers } \\
\text { - Attended educational sessions }\end{array}$ & $\begin{array}{l}\text { - Actively initiated tool usage } \\
\text { - Created and regularly updated 'CF Admission Board' with } \\
\text { pending admits } \\
\text { - Reviewed improvements at weekly meetings }\end{array}$ \\
\hline Pulmonary physicians & $\begin{array}{l}\text { - Participated in focus groups on workflow } \\
\text { - Attended educational sessions }\end{array}$ & $\begin{array}{l}\text { Participated in focus groups on new processes } \\
\text { - Followed regular updates on improvement } \\
\text { - Attended educational sessions on new interventions }\end{array}$ \\
\hline Fellows & $\begin{array}{l}\text { - Participated in focus group on QI and proposed processes } \\
\text { - Attended educational session }\end{array}$ & $\begin{array}{l}\text { - Participated in focus group on new process } \\
\text { - Attended educational session on new improvements }\end{array}$ \\
\hline Residents & $\begin{array}{l}\text { Participated in focus groups } \\
\text { - Attended educational sessions }\end{array}$ & $\begin{array}{l}\text { - Actively used the tool } \\
\text { Attended ongoing educational sessions, for new teams and new } \\
\text { improvements } \\
\text { - Participated in focus groups on new process }\end{array}$ \\
\hline Nurses & - Attended educational sessions & - Participated in focus groups on resultant process \\
\hline
\end{tabular}

below the mean was indicative of special cause variation and was equivalent to $\mathrm{p}<0.01 .{ }^{13}$ Statistical significance was also measured using a two-sample t test, with $\mathrm{p}<0.05$ indicating significance. Each PEx admission was treated as an independent event, even when patients returned for multiple hospitalisations. Our primary outcome of interest was defined as the time from arrival on the inpatient unit until the initial order and administration of first intravenous antibiotics. Baseline data was obtained from the 2 years before intervention $(\mathrm{n}=174)$ with continuous monitoring after interventions via I-chart. Tool usage was monitored via chart review and direct observation to assess adherence to the intervention. CHW Institutional Review Board approved the project.

\section{Results}

The handoff tool was implemented for all admissions beginning in September 2011. Multiple plan-dostudy-act cycles (PDSA) were used to improve workflow and the tool itself. Feedback was obtained through focus groups with end users and with regular review by our workgroups. One barrier identified early on was the physical transfer of the paper tool from the outpatient clinic to the inpatient team, a process which was time consuming and had limited reliability. To address this, an electronic version of the handoff tool was devised which could be completed and transmitted electronically. This was implemented 3 months after initial intervention and allowed for more efficient completion and improved reliability.

The disruption of clinic and hospital workflow due to unexpected CF admissions was identified as another cause of delay. The inpatient and outpatient teams felt that this could be mitigated if admissions could be planned for in advance. Although many CF hospitalisations are unexpected, CF outpatient providers can often anticipate which patients are at high risk for PEx and admission. To address this, a CF admission whiteboard was introduced in the pulmonary office. Possible admissions identified at our weekly $\mathrm{CF}$ centre meetings are posted here to alert inpatient pulmonary staff and allow for earlier preparation. Feedback from the CF team and pulmonary service showed that using the whiteboard improved preadmission planning, medication decisions, and facilitated a more efficient admission process.

The interventions led to a significant decrease in the time to antibiotic order from $3.01 \mathrm{~h}$ to $1.25 \mathrm{~h}(\mathrm{n}=88$, $\mathrm{p}<0.001$, figure 2). Accompanying this was a significant improvement in antibiotic administration time from $5.87 \mathrm{~h}$ to $4.41 \mathrm{~h}(\mathrm{p}<0.001)$. Through these changes we sustained a shift in our process for 14 months (figure 2), with expected variability throughout the year. Smaller training sessions were completed regularly for new residents and pulmonary teams. There was excellent engagement and involvement by all end users. Tool usage varied from $75 \%$ to $100 \%$ initially, with adherence stabilising at 100\% 8 months post intervention.

The resultant change in workflow and culture allowed for the immediate ordering of these treatments upon arrival on the unit (figure 1B). Focus groups with the paediatric house staff showed that residents found the workflow easier than before and still felt ownership of the patient. They felt that receiving a predetermined choice of antibiotics did not decrease their understanding of CF care. Feedback from parents in focus groups showed that they 


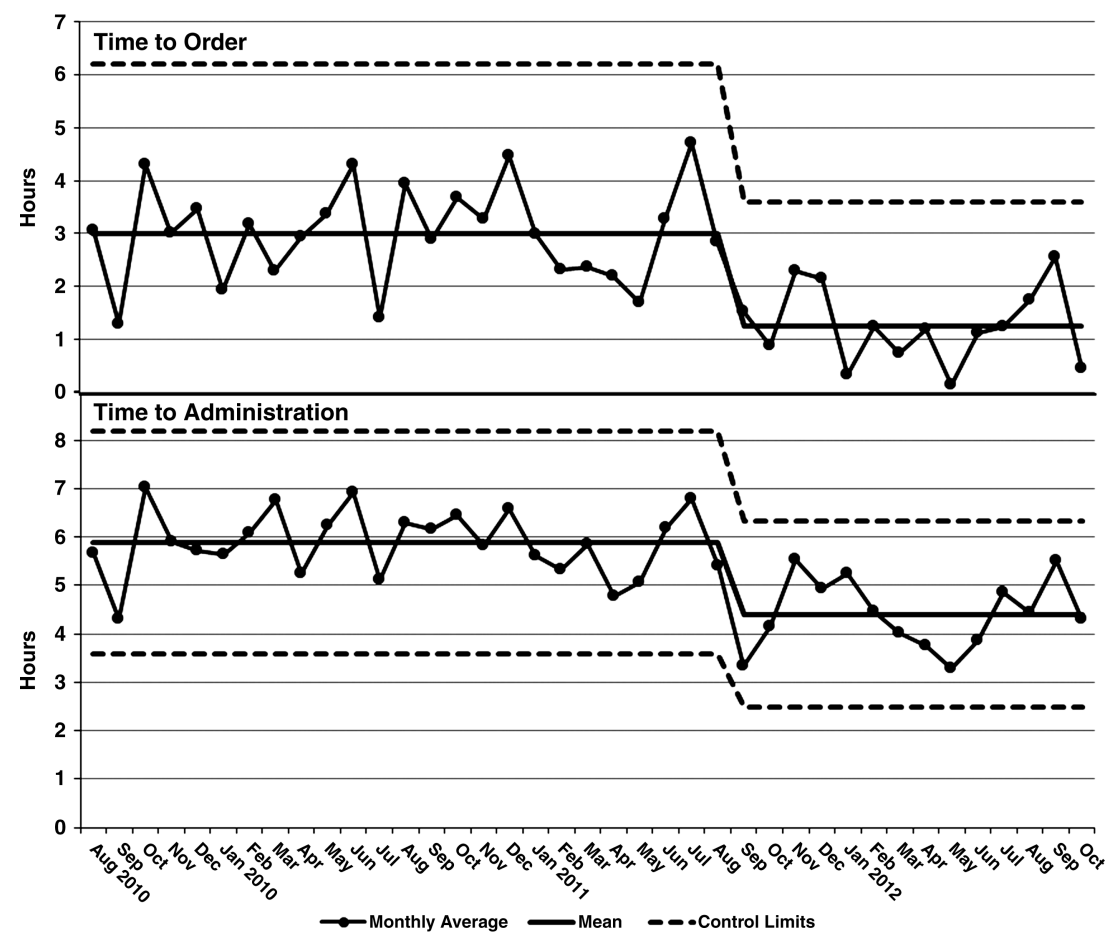

Figure 2 I-chart showing mean time from arrival on pulmonary unit until antibiotic order is placed and subsequently administered. Means and control limits shown for baseline and after main intervention.

perceived the process to be smoother and felt less anxious about the wait for antibiotic initiation. Periodic chart reviews for safety were completed to ensure that the correct antibiotics were ordered as recorded on the tool, with no adverse events identified. Overall, following the introduction of this handoff tool there has been a sustainable shift in the ordering process and an expectation that orders be placed earlier. Starting 14 months after intervention, CHW transitioned to a system-wide electronic health record and our next step is the transition and integration of the tool and our workflow into this new system.

\section{CHOA INITIATIVE TO STANDARDISE AND IMPROVE MULTIDISCIPLINARY INPATIENT TREATMENT OF CF PEX}

\section{Context}

The Emory Paediatric CF programme on the Egleston campus of Children's Hospital of Atlanta is a large academic programme that cares for about 250 patients. There are 100-130 CF PEx admissions at this site each year.

Transitions in our CF centre leadership and a relocation of all paediatric admissions to a single location led to a review of inpatient staff training and care processes. Our overall goal was to achieve a uniform standard of excellence and improve the outcomes of CF PEx treatment. Our specific aims included decreasing the proportion of admitted patients who do not return to baseline lung function at treatment end and to improve their weight gain.

\section{Methods and interventions}

Similar to CHW, a multidisciplinary steering committee was assembled that included leaders from hospital administration, medicine, nursing, respiratory therapy (RT), nutrition, physical therapy, social work, child life, and pharmacy, as well as a family council representative to help maintain a patient-centred and family centred approach. In addition, discipline-specific workgroups were also organised. CF care providers worked together to standardise care as much as possible, creating a standard order set and inpatient treatment algorithm. Other work groups were charged with planning how to implement care processes in a uniform manner. Work groups accomplished their tasks in various ways, including benchmarking visits to outside institutions to learn of others' successes and systematic re-education of staff in specific techniques such as ACT. Discipline-specific interventions to promote consistent delivery of therapies are described in table 2.

To educate staff on the rationale of the programme and allow them to maximally contribute to patient educational and therapeutic efforts, an annual ' $\mathrm{CF}$ Academy' was initiated. This day-long seminar, primarily aimed at the inpatient staff, is led by the CF team to educate on the principles and goals of CF treatment. The programme helped to increase awareness and improve consistency, and also taught the 
Table 2 Improvement steps at Children's Hospital of Atlanta

\begin{tabular}{|c|c|c|}
\hline & Planning Phase & Implementation Phase \\
\hline Multidisciplinary team & $\begin{array}{l}\text { - Participated in QI Workgroups } \\
\text { - Established 'CF Academy' }\end{array}$ & $\begin{array}{l}\text { - Attended weekly CF team meetings } \\
\text { - Attended monthly QI planning meetings } \\
\text { - Fostered a culture and interest in QI }\end{array}$ \\
\hline $\begin{array}{l}\text { Physicians and nurse } \\
\text { practitioners }\end{array}$ & $\begin{array}{l}\text { Established a consistent treatment plan } \\
\text { Created a standardised order set } \\
\text { - Created outpatient to inpatient handoff form }\end{array}$ & $\begin{array}{l}\text { Attended weekly inpatient multidisciplinary meetings } \\
\text { Used new forms, tools and plans } \\
\text { - Lent overall support for QI efforts } \\
\text { Created standard note and discharge summary } \\
\text { templates } \\
\text { - Respected and did not interrupt ACT sessions }\end{array}$ \\
\hline Nursing & $\begin{array}{l}\text { - Reviewed nursing activities and educational materials } \\
\text { used at other institutions } \\
\text { - Established a 'credentialed' group of core CF nurses }\end{array}$ & $\begin{array}{l}\text { - Met with family on admission to discuss programme } \\
\text { and create schedule } \\
\text { - Coordinated the schedule of all activities and therapies } \\
\text { - Acted as patient advocate and educator throughout } \\
\text { - Awarded patient tokens for observing schedule }\end{array}$ \\
\hline Respiratory Therapy & $\begin{array}{l}\text { - Performed a benchmarking visit to a leading CF centre } \\
\text { Developed standardised approaches to ACT } \\
\text { - Trained all therapists on standard ACT techniques }\end{array}$ & $\begin{array}{l}\text { - Attended patients on schedule } \\
\text { Maintained standardised performance and teaching of } \\
\text { ACT } \\
\text { - Posted sign at door during ACT to prevent } \\
\text { interruptions } \\
\text { - Awarded patient tokens for good participation in ACT }\end{array}$ \\
\hline Nutrition & - Developed menu with caloric content of food & $\begin{array}{l}\text { - Educated and gave advice for high caloric intake } \\
\text { - Worked with patients on calorie counts } \\
\text { - Awarded patient tokens for good meal intake } \\
\text { - Posted daily weight charts in rooms }\end{array}$ \\
\hline Physical therapy & $\begin{array}{l}\text { Developed measurement strategy } \\
\text { - Standardised therapy visits }\end{array}$ & $\begin{array}{l}\text { Set individualised plan } \\
\text { - Held PT sessions } 5 \text { days/week as scheduled } \\
\text { Assigned unsupervised weekend activities } \\
\text { - Awarded patient tokens for participation in therapy } \\
\text { activities }\end{array}$ \\
\hline Child life & - Established repository of prizes to be awarded for tokens & $\begin{array}{l}\text { - Supervised token-based incentive programme for } \\
\text { patients } \\
\text { - Awarded prizes at end of stay }\end{array}$ \\
\hline Patient and family & - Participated in workgroups and planning & $\begin{array}{l}\text { - Established schedule with nurses on admission } \\
\text { - Received tokens for good participation in programme } \\
\text { - Provided feedback }\end{array}$ \\
\hline
\end{tabular}

ACT, airway clearance therapy; CF, cystic fibrosis; QI, quality improvement.

value of each clinician's role and helped to foster an interest in improving the quality of care.

Nursing played a central role in coordinating the process. They worked with the other practitioners and the patients and families in establishing an explicit schedule for all therapeutic activities (including meals and school) to ensure that patients understood and were available to consistently receive all intended treatments. All treatment providers (including physicians) were expected to observe and respect the schedule for visits. Additionally, to increase family and patient involvement in care, a token-based incentive system was devised. Patients would receive tokens as a reward for on-time wake-up, observing curfew and active participation in all scheduled therapeutic activities. This was overseen by child life, with tokens being redeemed for prizes at the end of the hospitalisation.

Outcomes were studied and interpreted as described in the CHW methods above. Our primary outcome of interest was the likelihood of forced expiratory volume in $1 \mathrm{~s}$ (FEV1) returning to within 90\% of baseline by the end of the hospitalisation. Baseline FEV1 was defined as the maximum FEV1\% predicted recorded either since the last admission for treatment of PEx (including those obtained while hospitalised) or in the previous 12 months, whichever time period was shorter. This was compared with the maximum FEV1\% predicted measured during the hospitalisation of interest. Baseline data was obtained from the year prior to intervention with continuous monitoring after interventions via P-chart. Process measures were monitored via associated run chart, with baseline data obtained when possible and continuous monitoring after intervention. Emory University Institutional Review Board approved the project.

\section{RESULTS}

The programme was planned during the second half of 2008 and implemented in early 2009. We tracked FEV1 outcomes of 548 pulmonary exacerbations in children between the ages of 6 years and 21 years beginning with the baseline year of 2008 up to April 2013.

Multiple process measures were used to track disciplinespecific improvements and adherence, including: 
- Nursing (figure 3A,B): Measures of schedule adherence showed that waking of patients on time for morning care seemed to plateau by the $3 \mathrm{rd}$ month, and remained steady at about 93\%. Curfew adherence took 4 months to reach a stable plateau of about $88 \%$.

- Nutrition (figure 3C,D): There was notable difficulty with consistently and promptly getting enzymes to patients with meals, as hospital interpretation of regulations required that nurses dispense enzymes following the delivery of each meal. We had particular difficulty with lunchtime, which took about 5 months to level out at an average rate of $80 \%$.

- RT (figure 3E,F): During the planning and early implementation phase, $100 \%$ of therapists were refreshed on ACT techniques. The use of Huff cough during therapy sessions was particularly emphasised and reached a steady state of about $89 \%$ by the second half of the implementation period. Performance of 4 times a day ACT was documented $96 \%$ of the time after implementation.

- Child life/patient: A stable average of $85 \%$ of possible tokens was awarded during the monitoring period.

We retrospectively noted that the average weight gain during hospitalisation of patients whose body
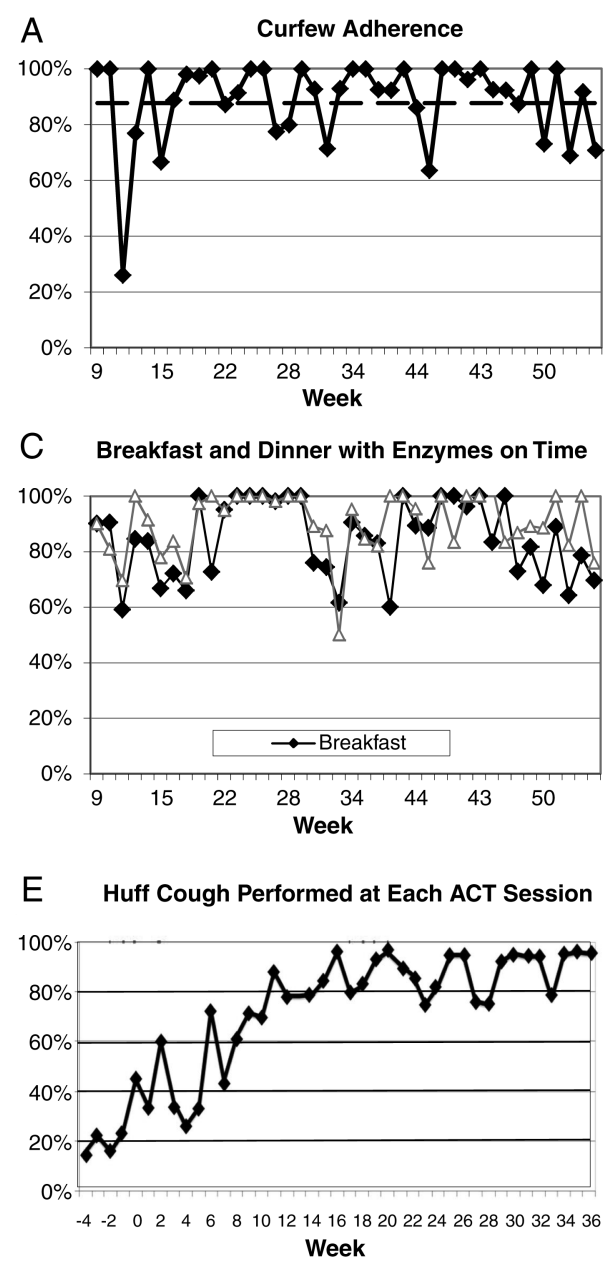

mass index (BMI) was less than the 10th centile rose from $4.5 \%$ at baseline to $8.4 \%$ during the programme $(\mathrm{p}=0.10)$. The average weight gain of those whose BMI was greater than the 50th centile rose from 1.1\% to $4.4 \%(p=0.04)$, however increased weight gain was not statistically significant for the group as a whole.

As shown in figure 4, prior to the initiation of our programme an average of $31 \%$ of patients failed to recover their FEV1 to within $90 \%$ of their baseline; following implementation of the programme, that percentage successfully dropped to $18 \%$. This change was indicative of special cause variation using conventional statistical process control methods, and has been sustained for about 3 years at this point. We found a similar trend when recovery to $100 \%$ of baseline FEV1 was measured, with a decrease from 66\% unsuccessful to $59 \%$, but this measure did not attain criteria for formal special cause variation.

\section{DISCUSSION}

Inpatient $\mathrm{CF}$ care is a complex and multifaceted process that requires numerous therapies and coordination among multiple physicians, therapists, nurses,
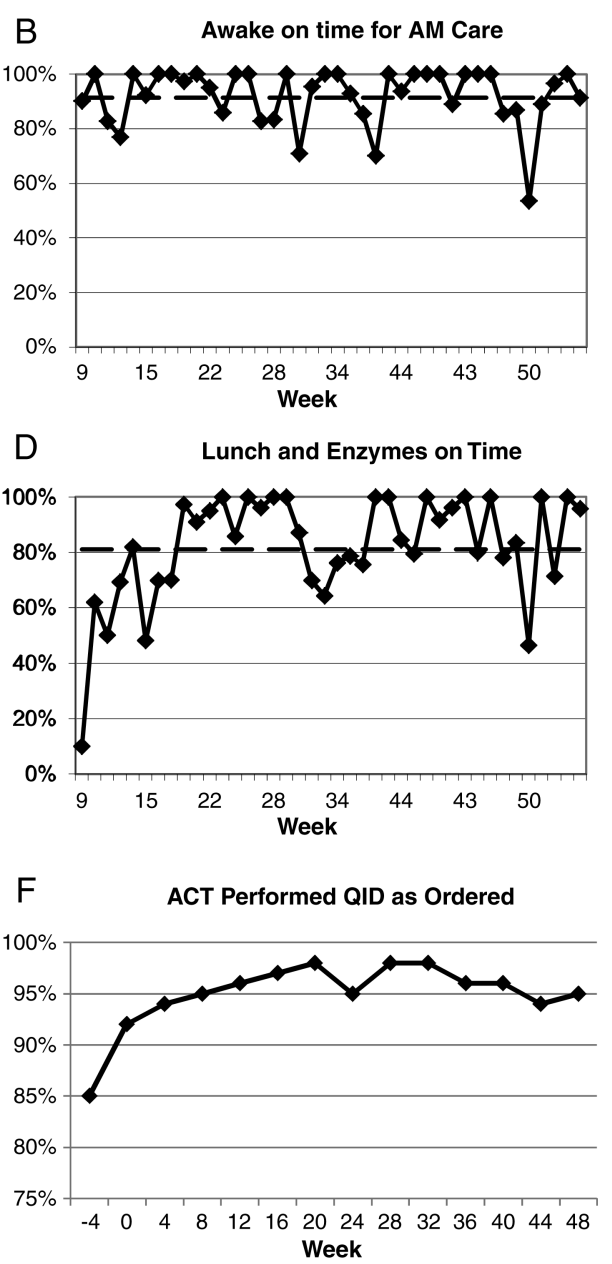

Figure 3 Run charts showing process measures tracked including nursing (A, B), nutrition (C, D) and respiratory therapy (E, F). Time shown in weeks from intervention. 


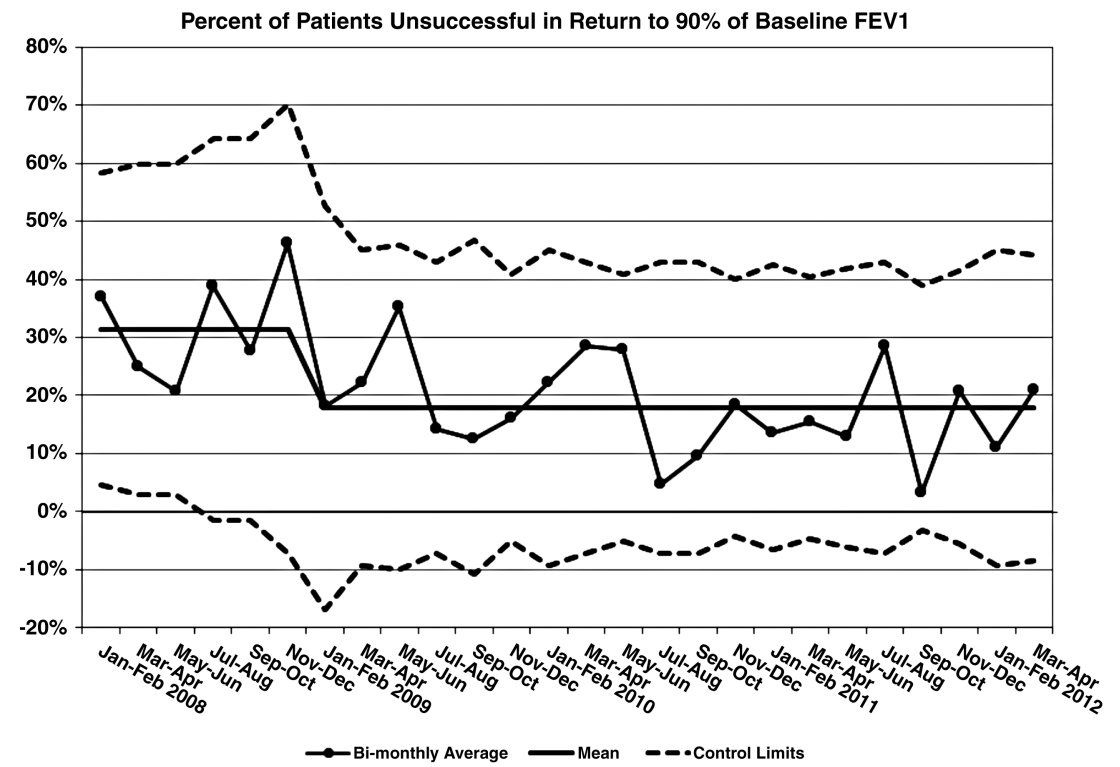

Figure 4 P-chart showing the per cent of patients unsuccessful in return to $90 \%$ of baseline FEV1\% predicted after treatment. Means and control limits shown for baseline and after main intervention.

nutritionists and other clinical staff. In addition, most CF centres are academic institutions where attention to trainee education is an important but complicating priority. The QI initiatives described in this report focused on standardisation and coordination of care to improve outcomes and the patient experience. At $\mathrm{CHW}$, the use of a written handoff tool to improve the efficiency of physician hand offs successfully decreased the time to initial antibiotic order by more than $50 \%$, resulting in a sustained decrease in the time to administration by $25 \%$. The handoff tool allowed a safe change in our culture and expectations, while supporting the educational system. At CHoA, a multidisciplinary team initiative to standardise care, augmented by a patient incentive programme, led to a decrease of over $40 \%$ in the per cent of patients who failed to return their FEV1 to within $90 \%$ of baseline. Similar techniques and QI methods were used to implement successful changes at both CF centres, with key strategies shown in box 1 .

Little literature exists on QI initiatives in CF inpatient care, in part because only relatively large CF programmes have enough hospitalisations to measure impact. QI reports in emergency and intensive care settings have shown improvements in timeliness to treatments and specifically, initiation of antibiotics, ${ }^{14} 15$ as at CHW. These studies similarly imparted change by decreasing variation ${ }^{14}$ and standardising therapy practices. ${ }^{15}$ Studies in multiple disciplines have shown that team-based training interventions can improve staff communication and satisfaction, ${ }^{12} 16$ one of the major initiatives at CHoA. Central to all these studies is the inclusion of multiple team members in the planning and development of the training and education regimens. There is also increasing evidence on the importance of continuous monitoring in QI methodology ${ }^{11-14}{ }^{16}$ At both centres continuous monitoring of outcomes helped to identify barriers, plan improvements, and monitor the outcomes and success of interventions.

The success of our projects also depended on their fitting into the systems and infrastructure at each respective institution. At $\mathrm{CHW}$ the well-established outcomes and QI culture helped to ease the

Box 1 Strategies for Successful Improvement of Inpatient Care

Creation of a multidisciplinary quality improvement (QI) team with key personnel

- Regular meetings and feedback from the team

- Specific roles and goals for team members

- Active engagement of administration and all personnel in the QI process

- Patients and families taking an active role in improvement process

- Detailed review of current processes and planning of improvements

- Creation and usage of standardised tools for care coordination, such as a written handoff tool

- Discipline-specific training sessions for all end users and key personnel

- System-wide education efforts on care and QI, such as the 'CF Academy'

- Continuous monitoring of improvements after the interventions

- Reporting of improvement barriers and successes to all team members

- Using lessons learned from each project towards future improvement efforts 
development and incorporation of these tools into our standard workflow. While this culture was less well established at $\mathrm{CHoA}$, goals at both centres were accomplished by securing active support from senior leaders and managers in multiple disciplines. This involvement facilitated the trial, implementation, measurement and embracement of multiple attempts to improve the status quo. Although independent initiatives, much of the improvement planning and key strategies were the same for both our institutions. Approaches which were crucial to our combined successes are shown in box 1 .

Obstacles at both institutions included the need to coordinate multiple practitioners, gain acceptance of standardised approaches and find common ground regarding competing priorities. The engagement of multidisciplinary workgroups allowed the QI teams to anticipate and address these obstacles. Significant effort was put into the creation of incentives for adoption of these changes for patients and providers alike. This includes the building of educational payback into the written handoff tool at $\mathrm{CHW}$ and the innovative token programme at $\mathrm{CHoA}$. Limitations to the generalisability of these interventions include the very specific patient population and the unique nature of our institutions, as large free-standing academic children's hospitals. The significant support required from administration and end users can also be a barrier to instituting these types of changes. Despite these barriers, similar concepts and projects can effectively be applied to multiple different disease states or admission types.

There is concern that trainees' use of algorithms and standard care pathways may impede learning and that a loss of autonomy may decrease medical education and job satisfaction. ${ }^{17}$ However, current medical education must balance teaching priorities with expectations for the practice of evidence-based medicine, prevention of delays in treatment and avoidance of medical errors. ${ }^{18}$ For example, at both institutions the admitting physicians determined antibiotic choice without first allowing residents to formulate their own plan. By promoting education in other ways and building payback into the system with improved workflow, there was consistent engagement and no decrease in perceived education.

While always a concern, at this point there is no evidence of weakening of the projects' effects over time. The resultant cultural shift and new expectations fostered by these initiatives have caused sustainment of the changes. In both cases, we believe that this will persist due to the clear advantages of these procedures, the ease of the new workflow, and the empowerment of patient advocacy groups and staff teams to ensure these activities are appropriately prioritised. Future directions include the integration of a handoff tool into an electronic health record (at
CHW) and increased efforts to address patient gaps in CF knowledge (at CHoA).

In conclusion, efforts to improve care coordination and communication are extremely important for optimising inpatient care. We have shown that this type of intervention can improve the experiences and outcomes of patients with CF admitted for treatment of pulmonary exacerbations. Written handoff tools like the one devised at $\mathrm{CHW}$ and regular schedules and treatment regimens as introduced at $\mathrm{CHoA}$ are examples of innovations that can be adapted to many different institutions and disease states. This approach is particularly important at an academic institution where multiple providers at various levels of training are an essential part of the system, and communication breakdown is common. By increasing end user and family involvement and bringing together staff teams, interventions like these can be sustained over longer periods of time. Focused improvement efforts on physician communication and standardised care coordination can thereby improve the timeliness and outcomes of pulmonary exacerbation care.

Acknowledgements The inpatient improvements described at $\mathrm{CHW}$ were only successful due to the dedication, efforts and leadership of the CF centre care team and QI workgroup including Tami Miller, Alice Stonek, Amanda Quesnell, Dr Juan P Ruiz, Brittani Clark, Emma Kennedy, Sue Malaney and Mary Ellen Freeman. Thanks to Dr Lynn D'Andrea, pulmonary division head, for support of these initiatives and manuscript preparation. Process improvement and statistics support from the CHW Outcomes Department and the National Outcomes Center, including Kristyn Maletta, Kyle Steffen and Bixiang Ren. "Breathe In, Blast Off", the inpatient CF improvement programme at CHoA, would not have gotten off the ground without the assistance of our steering committee (Tim Coons, Nikki O'Hara, Traci Antes, Clara Hopkins, Lucy Wynne, Tracy Frost, Liz Revilla, Kim Williamson, Tricia Easley, Tamila Paules, Lindsay Damron, Brandy Jones and Jamie Shropshire) and the hard work and dedication of our Workgroup Leads (Tamila Paules, Fawn Hastay, Liz Revilla, Lucy Wynn, Kim Williamson) and the rest of the CHoA Egleston inpatient staff, especially Maegan Pharis, Debra Samuel, Christine Middour and Megan Pankow. Thanks also to all the families of children with CF who kindly and patiently showed us the many opportunities for improvement and were a vital part of the successes in both of our centres.

Contributors NJA and MSS contributed equally to the creation and editing of this manuscript and led the separate projects at CHW (NJA) and CHoA (MSS). DRQ, CMW-K, and JEN aided in the project design, analysis and writing for the efforts at $\mathrm{CHW}$ and in editing the manuscript as a whole.

Funding Funded in part by quality improvement grants from the Cystic Fibrosis Foundation at CHW (ANTOS11QI0) and CHoA (SCHECH09QI0).

Competing interests None.

Ethics approval Children's Hospital of Wisconsin IRB and Emory University IRB, respectively.

Provenance and peer review Not commissioned; externally peer reviewed.

\section{REFERENCES}

1 Cystic Fibrosis Foundation. Cystic fibrosis foundation patient registry: 2011 annual data report. Bethesda, MD: Cystic Fibrosis Foundation, 2012. 
2 Hegarty M, Macdonald J, Watter P, et al. Quality of life in young people with cystic fibrosis: effects of hospitalization, age and gender, and differences in parent/child perceptions. Child Care Health Dev 2009;35:462-8.

3 Schreyogg J, Hollmeyer H, Bluemel M, et al. Hospitalisation costs of cystic fibrosis. PharmacoEconomics 2006;24:999-1009.

4 Ferkol T, Rosenfeld M, Milia CE. Cystic fibrosis pulmonary exacerbations. J Pediatr 2006;148:259-64.

5 Waters V, Stanojevic S, Atenafu EG, et al. Effect of pulmonary exacerbations on long-term lung function decline in cystic fibrosis. Eur Respir J 2012;40:61-6.

6 Sanders DB, Hoffman LR, Emerson J, et al. Return of FEV1 after pulmonary exacerbation in children with cystic fibrosis. Pediatr Pulmonol 2010;45:127-34.

7 Visse M, Abma T, Oever H, et al. Perceptions of hospital admission in patients with cystic fibrosis. J Hospital Adm 2013;2:54-64.

8 Flume PA, Mogayzel PJ, Robinson KA, et al. Cystic fibrosis pulmonary guidelines: treatment of pulmonary exacerbations. Am J Respir Crit Care Med 2009;180:802-8.

9 Schechter MS, Gutierrez HH. Improving the quality of care for patients with cystic fibrosis. Curr Opin Pediatr 2010;22:296-301.

10 Peterson TH, Teman SF, Connors RH. A safety culture transformation: its effects at a children's hospital. J Patient Saf 2012;8:125-30.
11 Agency for Healthcare Research and Quality. Closing the quality gap: a critical analysis of quality improvement strategies. Rockville, MD: Agency for Healthcare Research and Quality, 2004.

12 Bunnell CA, Gross AH, Weingart SN, et al. High performance teamwork training and systems redesign in outpatient oncology. BMJ Qual Saf 2013;22:405-13.

13 Benneyan JC, Lloyd RC, Plsek PE. Statistical process control as a tool for research and healthcare improvement. Qual Saf Health Care 2003;12:458-64.

14 Bookman LT, Troy R, McCaffrey R, et al. Using qualityimprovement methods to reduce variation in surfactant administration. Qual Saf Health Care 2010;19.

15 Volpe D, Harrison S, Damian F, et al. Improving timeliness of antibiotic delivery for patients with fever and suspected neutropenia in a pediatric emergency department. Pediatr 2012;130:e201-10.

16 Brodsky D, Gupta M, Quinn M, et al. Building collaborative teams in neonatal intensive care. BMJ Qual Saf 2013;22:374-82.

17 Williams GC, Deci EL. The importance of supporting autonomy in medical education. Ann Intern Med 1998;129:303-8.

18 Institute of Medicine (U.S.) Committee on Quality of Health Care in America. Crossing the quality chasm: a new health system for the 21st century. Washington, DC: National Academy Press, 2001. 